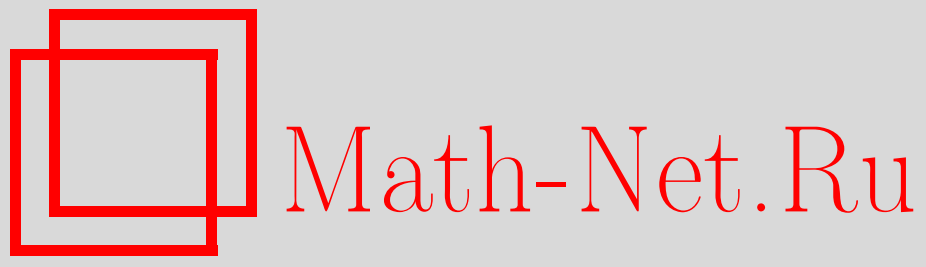

В. С. Молдавский, Модули эллиптических кривых и числа вращения диффеоморфизмов окружности, Функи. анализ и его прил., 2001, том 35, выпуск $3,88-91$

DOI: https://doi.org/10.4213/faa265

Использование Общероссийского математического портала MathNet.Ru подразумевает, что вы прочитали и согласны с пользовательским соглашением

http://www.mathnet.ru/rus/agreement

Параметры загрузки:

IP: 3.89 .197 .203

26 апреля 2023 г., 16:34:24

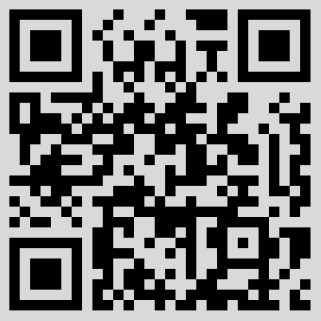




$$
\begin{aligned}
& \lambda_{k} \sigma_{k+1} \sigma_{k}=\sigma_{k} \lambda_{k+1}, \sigma_{k} \sigma_{k+1} y_{k}=y_{k+1} \sigma_{k}, \sigma_{k} \lambda_{k}=\lambda_{k+1} \sigma_{k} \sigma_{k+1}, y_{k} \sigma_{k}=\sigma_{k+1} \sigma_{k} y_{k+1}, \\
& \lambda_{k} \sigma_{k}=\lambda_{k}, \quad \sigma_{k} y_{k}=y_{k} . \quad \square
\end{aligned}
$$

Любое бесконечное плетение можно преобразовать в 3-диаграмму, поставив его оси $\mathbb{R}_{+}$вертикально, приклеив к полученному нижнему краю $\mathbb{R} \times 0$ две полуплоскости и стянув все перекрестки и 3 -валентные вершины на ось $\mathbb{R} \times 0$. Это задает вложение полугруппы бесконечных плетений Sit в полугруппу Stg. В частности, образующим соответствуют элементарные слова: $u_{k}=d_{3}^{k} c_{3} b_{3}^{k-1}$, $v_{k}=d_{3}^{k-1} a_{3} b_{3}^{k}, \sigma_{k}=d_{3}^{k-1} b_{2} d_{3} d_{2} b_{3}^{k}, \lambda_{k}=d_{3}^{k-1} e_{3} b_{3}^{k}, y_{k}=d_{3}^{k} f_{3} b_{3}^{k-1}$. Любое сбалансированное слово разлагается на элементарные аналогично тому, как это делается в [12], т. е. такое слово определяет некоторое бесконечное плетение. Поскольку каждый заузленный граф представляется сбалансированным словом, ему можно поставить в соответствие бесконечное плетение. При этом изотопным заузленным графам отвечают изотопные бесконечные плетения. Чтобы закончить доказательство теоремы 2, осталось вывести соотношения (7)-(15) полугруппы Sit из соотношений (1)-(6) полугруппы Stg. Разложение на элементарные слова и вывод соотношений аналогичны соответствующим разложениям в [12].

Автор выражает благодарность своему научному руководителю профессору В. М. Бухштаберу за поддержку и доценту И. А. Дынникову за внимание к работе.

\title{
ЛИТЕРАТУРА
}

1. Дынников И. А. Функц. анализ и его прил., 33, вып. 4, 25-37 (1999). 2. Дынников И. А. Функц. анализ и его прил., 34, вып. 1, 29-40 (2000). 3. Kauffman L. Trans. Amer. Math. Soc., 311, No. 2, 697-710 (1989). 4. Jonish, D., Millett, K. C. Trans. Amer. Math. Soc., 327, No. 2, 655-702 (1991). 5. Taniyma K. Topology, 33, No. 3, 509-523 (1994). 6. Litherland R. Math. Proc. Cambridge Philos. Soc., 106, No. 1, 95-106 (1989). 7. Stenford T. Topology, 35, No. 4, 1027-1050 (1996). 8. Франк-Каменецкий Самая главная молекула (Библиотечка «Квант»). 9. Альбертс и др. Молекулярная биология клетки, т. 1. 10. Шилл Г. Катенаны, ротоксаны и узлы. Мир, М., 1973. 11. Simon J. Topology, 25, 229-235 (1986). 12. Дынников И. А. Конечно-определенные полугруппы и группы в теории узлов. Труды МИАН, т. 231.

Московский государственный университет

Поступило в редакцию 22 июня 2000 г.

УДК 517.535.2

\section{Модули эллиптических кривых и числа вращения диффеоморфизмов окружности}

\author{
(c) 2001. В. С. МолдАвСКий
}

Комплексное возмущение аналитического диффеоморфизма окружности - это биголоморфное отображение, фундаментальная область которого - эллиптическая кривая. В. И. Арнольд предположил, что модуль этой кривой стремится к числу вращения исходного диффеоморфизма при стремлении к нулю параметра 
возмущения. В работе доказывается гипотеза Арнольда для аналитически эквивалентных повороту диффеоморфизмов окружности. Доказательство проводится с помощью теории квазиконформных отображений (см. [2]).

Пусть $F: S^{1} \rightarrow S^{1}$ - аналитический диффеоморфизм окружности. Поднимем $F$ до диффеоморфизма прямой $\widetilde{F}: \mathbb{R} \rightarrow \mathbb{R}$. Обозначим через $F_{\alpha}$ отображение $\widetilde{F}+i \alpha$ нижней границы полосы $\Pi_{\alpha}=\{z \in \mathbb{C}: 0 \leqslant \operatorname{Im} z \leqslant \alpha\}$ на верхнюю. Отождествим точки из $\Pi_{\alpha}$, различающиеся на целое действительное число; у полученного цилиндра склеим основания по отображению $F_{\alpha}$. Получится эллиптическая кривая $T_{\alpha}$ с комплексной структурой, унаследованной от накрывающей полосы. Она биголоморфно эквивалентна $\mathbb{C} /\left(\mathbb{Z}+b_{\alpha} \mathbb{Z}\right)$, где $\operatorname{Im} b_{\alpha}>0$ и $b_{\alpha}$ определено с точностью до прибавления целого числа. Положим $\mu_{\alpha}:=b_{\alpha} \bmod \mathbb{Z}$ и будем называть $\mu_{\alpha}$ модулем эллиптической кривой $T_{\alpha}$. Оказывается, что в достаточно общем случае модули кривых $T_{\alpha}$ стремятся к числу вращения диффеоморфизма $F$. Точнее, верен следующий результат:

ТЕОРема 1. Пусть F- аналитически эквивалентный повороту на $2 \pi \mu$ аналитический диффеоморфизм окружности. Тогда модули $\mu_{\alpha}$ эллиптических кривых $T_{\alpha}$, построенных по отображению $F$, стремятся $\kappa \mu$ при $\alpha \rightarrow 0$.

Из теоремы Эрмана [3] вытекает

СледствиЕ. Если $F$ - аналитический диффеоморфизм окружности с числом вращения $\mu$, принадлежащим некоторому множеству полной меры, то $\mu_{\alpha} \rightarrow \mu$ при $\alpha \rightarrow 0$.

ПрЕДЛОЖЕНИЕ 1 [2]. Среди отображений плоскости с выколотыми точками 0, 1, $\tau_{1}, 1+\tau_{1}$ на плоскость с выколотымми точками 0, $1, \tau_{2}, 1+\tau_{2}$, $\operatorname{Im} \tau_{i}>0$, аффинное отображение имеет наименьшее квазиконформное отклонение, равное $e^{d\left[\tau_{1}, \tau_{2}\right]}$, где $d[\cdot, \cdot]$ - расстояние в метрике Лобачевского на верхней полуплоскости.

Лемма 1. Рассмотрим эллиптические кривые $T_{1}$ и $T_{2}, T_{i}=\mathbb{C} / \Gamma_{i}, \Gamma_{i}=\mathbb{Z}+$ $\tau_{i} \mathbb{Z}, \operatorname{Im} \tau_{i}>0$. Если существует K-квазиконформный гомеоморфизм $f: T_{1} \rightarrow$ $T_{2}$, то $d\left[\tau_{1}, \tau_{2}+m\right]<\ln K$ для некоторого изелого $\mathrm{m}$.

ДокАЗАтЕЛьство. Поднимем отображение $f$ до отображения $\tilde{f}: \mathbb{C} \rightarrow \mathbb{C}$ универсальных накрывающих. Линейными заменами в образе и прообразе добьемся того, чтобы $\tilde{f}\left(\Gamma_{1}\right)=\Gamma_{2}, \tilde{f}(0)=0, \tilde{f}(1)=1$. Тогда $\tilde{f}\left(\tau_{1}\right) \equiv \tau_{2}(\bmod \mathbb{Z})$. Пользуясь предложением 1 и учитывая, что квазиконформное отклонение отображения $\tilde{f}$ совпадает с квазиконформным отклонением отображения $f$, получим утверждение леммы.

ОсНОВНАЯ ЛЕммА. Пусть $T_{\alpha}$ - эллиптическая кривая из теоремь 1. Тогда существует квазиконформное отображение $T_{\alpha} \rightarrow T_{\alpha}^{0}=\mathbb{C} / \mathbb{Z}+(\mu+i \alpha) \mathbb{Z}$, показатель квазиконформности которого оценивается сверху величиной, не зависящей от $\alpha$, при всех $\alpha>0$ из некоторого полуинтервала $(0, \varepsilon]$.

Покажем, что из основной леммы следует теорема 1. По лемме 1 при $\alpha$, стремящемся к 0 , расстояние между $\mu_{\alpha}$ и $\mu+m+i \alpha$ остается меньшим константы $C$ в метрике Лобачевского для некоторого целого $m$, зависящего от $\alpha$, а значит, в евклидовой метрике расстояние между $\mu_{\alpha}$ и $\mu+m+i \alpha$ стремится к 0 ; отсюда следует, что $m=0$ для малых $\alpha$. Тогда $\left|\mu+i \alpha-\mu_{\alpha}\right| \rightarrow 0$ при $\alpha \rightarrow 0$, что и доказывает лемму.

Доказательство основной леммы проводится в несколько шагов. 
Шаг 1. Пусть $H(x)=x+h(x)-$ аналитическая замена, сопрягающая диффеоморфизм $\widetilde{F}$ с поворотом: $H \circ \widetilde{F} \circ H^{-1}=x+\mu$. Эта замена продолжается голоморфно в полосу $|\operatorname{Im} z| \leqslant \alpha_{0}$ для некоторого $\alpha_{0}>0$. Пусть $\alpha<\alpha_{0}$; тогда кривая $T_{\alpha}$ биголоморфно эквивалентна эллиптической кривой, полученной из криволинейной полосы $H\left(\Pi_{\alpha}\right)$ следующими отождествлениями: $z \equiv z+1$ для всех точек полосы и $H(x) \equiv H\left(F_{\alpha}(x)\right)$ для $x \in \mathbb{R}$. Последнюю склейку можно записать как $x \equiv G_{\alpha}(x)$, где

$$
G_{\alpha}(x)=H\left(\widetilde{F} \circ H^{-1}(x)+i \alpha\right) .
$$

Разлагая правую часть формулы (1) в ряд Тейлора по $\alpha$, получим

ПрЕДЛОЖЕНИЕ 2. Отображение (1) имеет вид

$$
G_{\alpha}(x)=x+\mu+i \alpha H^{\prime}\left(\widetilde{F} \circ H^{-1}(x)\right)+\alpha^{2} g(x, \alpha),
$$

где $g(x, \alpha)$ - аналитическая функция на $\mathbb{R} \times\left[0 ; \alpha_{0}\right]$, 1-периодическая по $x$.

ПрЕДЛОЖениЕ 3. Верхняя граница криволинейной полосы $H\left(\Pi_{\alpha}\right)$ является графиком функции $y=L_{\alpha}(x)$.

ДОКАЗАТЕЛЬСТВО. Положим $X_{\alpha}(x)=\operatorname{Re} G_{\alpha}(x)$ и $Y_{\alpha}(x)=\operatorname{Im} G_{\alpha}(x)$. Из предложения 2 следует, что

$$
X_{\alpha}(x)=x+\mu+\alpha^{2} \operatorname{Re} g(x, \alpha) .
$$

Значит, $X_{\alpha}^{\prime}>0$ при малых $\alpha, X_{\alpha}$ монотонна и, следовательно, обратима. Тогда $y=L_{\alpha}(x)=Y_{\alpha} \circ X_{\alpha}^{-1}(x)$. Нам понадобится явное выражение для $L_{\alpha}$ :

$$
L_{\alpha}=\alpha H^{\prime}\left(\widetilde{F} \circ H^{-1}(x-\mu)\right)+O\left(\alpha^{2}\right) .
$$

Следующие два шага переводят с помощью квазиконформного отображения криволинейную полосу $H\left(\Pi_{\alpha}\right)$ в прямолинейную полосу $\Pi_{\alpha}$, а склейку $x \rightarrow$ $G_{\alpha}(x)$ в стандартную склейку $x \rightarrow x+\mu+i \alpha$.

Шаг 2. Положим $\Phi_{\alpha}: \mathbb{C} \rightarrow \mathbb{C}, \Phi_{\alpha}(x+i y)=x+\left(\alpha / L_{\alpha}(x)\right) i y$. Согласно определению функции $L_{\alpha}, \Phi_{\alpha}\left(H\left(\Pi_{\alpha}\right)\right)=\Pi_{\alpha}$.

ПредложениЕ 4. Показатель квазиконформности отображения $\Phi_{\alpha}$ равномерно ограничен по а на отрезке $\left[0 ; \alpha_{0}\right]$.

ДокАЗАТЕЛЬСтво. Достаточно доказать, что показатель квазиконформности остается ограниченным при стремлении $\alpha$ к 0. Из (3) следует, что

$$
\Phi_{\alpha}(x+i y)=x+\frac{i y}{H^{\prime}\left(\widetilde{F} \circ H^{-1}(x-\mu)\right)}+i \alpha \varphi(x, \alpha) y,
$$

где $\varphi(x, \alpha)$ аналитична в $\mathbb{R} \times\left(0 ; \alpha_{0}\right]$. Семейство отображений плоскости $\Phi_{\alpha}$ стремится равномерно вместе с производными к отображению $\Phi_{0}, \Phi_{0}(x+i y)=$ $x+i y / H^{\prime}\left(\widetilde{F} \circ H^{-1}(x-\mu)\right)$, и, значит, квазиконформное отклонение отображения $\Phi_{\alpha}$ стремится к отклонению отображения $\Phi_{0}$, которое конечно в силу того, что $0<h_{1} \leqslant H^{\prime}(x) \leqslant h_{2}$ на $\mathbb{R}$. Предложение доказано.

Шаг 3. Рассмотрим отображение $S_{\alpha}: \mathbb{R} \rightarrow \mathbb{R}, S_{\alpha}=X_{\alpha}^{-1}(x)+\mu$. Имеем $S_{\alpha}(x)=$ $x+\alpha^{2} s(x, \alpha)$. Положим $\Psi_{\alpha}: \mathbb{C} \rightarrow \mathbb{C}, \Psi_{\alpha}(x+i y)=x+\alpha y s(x, \alpha)+i y$. В любой полосе конечной высоты, например $\Pi_{1}, \Psi_{\alpha}$ сходится вместе с производными к тождественному отображению при $\alpha \rightarrow 0$. Это доказывает

ПРЕДЛОЖеНИЕ 5. Показатель квазиконформности отображения $\Psi_{\alpha}$ стремится $\kappa 1$ при $\alpha \rightarrow 0$. 
Шаг 4. Композиция $\widetilde{R}_{\alpha}=\Psi_{\alpha} \circ \Phi_{\alpha}$ переводит склейку $z \rightarrow z+1$ в себя, а склейку $x \rightarrow G_{\alpha}(x)$ в $x \rightarrow x+\mu+i \alpha$ и потому опускается до отображения $R_{\alpha}: T_{\alpha} \rightarrow T_{\alpha}^{0}$. Показатель квазиконформности отображения $R_{\alpha}$ совпадает с показателем квазиконформности отображения $\widetilde{R}_{\alpha}$, который по предложениям 4 и 5 ограничен на полуинтервале $\left(0 ; \alpha_{0}\right]$. Отображение $R_{\alpha}-$ искомое. Основная лемма доказана.

\title{
ЛИТЕРАТУРА
}

1. Арнольд В. И. Дополнительные главы теории обыкновенных дифференциальных уравнений. Наука, М., 1978. 2. Альфорс Л. Лекции по квазиконформным отображениям. Мир, М., 1969. 3. Herman M.-R. Inst. Hautes Études Publ. Math., 49, 5-233 (1979).

Московский государственный университет

Поступило в редакцию 5 июня 2000 г.

УДК 517.55

\section{К проблеме Рубеля-Тейлора о представлении мероморфных функций ${ }^{*}$}

\author{
(c) 2001. Б. Н. ХАБиБУллин
}

Пусть $f$ - мероморфная функция на $\mathbb{C}^{n}$ (мы используем изображение $f \in$ Mer) и $f=g_{f} / h_{f}$ - ее каноническое представление как частного целых функций $g_{f}$ и $h_{f}$, взаимно простых в каждом кольце ростков голоморфных в точке из $\mathbb{C}^{n}$ функций [1]. Характеристическую функцию функции $f \in$ Mer определим формулой

$$
u_{f}=\max \left\{\ln \left|g_{f}\right|, \ln \left|h_{f}\right|\right\} \quad \text { или } \quad u_{f}=\ln \left(\left|g_{f}\right|^{2}+\left|h_{f}\right|^{2}\right)^{1 / 2} .
$$

Пусть $\mathrm{E}=\left(L_{1}, \ldots, L_{k}\right)-$ набор комплексных векторных подпространств в $\mathbb{C}^{n}$ и $\mathbb{C}^{n}=L_{1} \oplus \cdots \oplus L_{k}-$ их прямая сумма. Пусть

$$
z=w_{1}+\cdots+w_{k}, \quad w_{p} \in L_{p}, p=1, \ldots, k,
$$

- единственное представление вектора $z \in \mathbb{C}^{n}, r_{p}=\left|w_{p}\right|$ - евклидова норма точки $w_{p}$ и $\vec{r}=\left(r_{1}, \ldots, r_{k}\right)$.

Обозначим через $S_{p}$ единичную сферу в $L_{p}$, а через $d s^{(p)}$ элемент площади на $S_{p}, s^{(p)}\left(S_{p}\right)=1$.

Пусть $f \in$ Mer и в $(2) w_{p}=r_{p} \zeta_{p}, \zeta_{p} \in S_{p}$. Функция

$$
T_{\mathrm{E}}(\vec{r} ; f)=\int_{S_{1}} \cdots \int_{S_{k}} u_{f}\left(r_{1} \zeta_{1}, \ldots, r_{k} \zeta_{k}\right) d s^{(1)}\left(\zeta_{1}\right) \cdots d s^{(k)}\left(\zeta_{k}\right),
$$

где $\vec{r} \in \mathbb{R}_{+}^{k}, \mathbb{R}_{+}=\{a \in \mathbb{R}: a>0\}$, называется Е-характеристикой Неванлинны функции $f$. При $k=1$ (при $k=n$ ) это классическая характеристика Неванлинны через исчерпание по шарам [2-7] (соответственно по полидискам [5]). L-характеристика Неванлинны определена однозначно с точностью до постоянного

\footnotetext{
*Работа выполнена при финансовой поддержке РФФИ, проект 00-01-00770.
} 